\title{
The Effects of Trunk Cavity and Air-Gaps in the Acoustic Response of a Passenger Vehicle
}

\author{
Akın OKTAV \\ Vibration and Acoustics Laboratory \\ Department of Mechanical Engineering \\ Bogazici University \\ Bebek 34342 Istanbul, Turkey; e-mail: akin.oktav@boun.edu.tr, akinoktav@yahoo.co.uk
}

(received November 18, 2016; accepted March 28, 2017)

\begin{abstract}
The effects of additional cavities and air-gaps in the acoustic response of a passenger vehicle are investigated. It is observed that the cabin cavity and the trunk cavity of the passenger vehicle are connected through an aperture in the rear seat. In the trunk cavity of the vehicle, there are two more air-gaps which are designed as countermeasures to trunk lid slam noise. It is established that acoustic modes and acoustic eigenfrequencies of the vehicle are altered through the trunk cavity and its air-gaps. To develop an analytical solution, the actual acoustic cavity is simplified into a rectangular shape. In the analytical solution, the coupling of trunk and cabin cavities is considered. It is shown that the computational analysis results match well with the results of the analytical solution proposed. Further, the resonator effect of air-gaps present in the trunk cavity is examined.
\end{abstract}

Keywords: acoustic coupling; acoustic eigenfrequencies; acoustic modes; acoustic response; air-gap; cabin cavity; Helmholtz resonator; passenger vehicle; trunk cavity; wave number.

\section{Introduction}

The acoustic comfort of passenger vehicles is an important competition factor in the market. It strongly influences the customer satisfaction, especially in long term. To improve the acoustic comfort, vibro-acoustic analysis is performed before and after the vehicle launch (FERREIRA et al., 2016). For a robust analysis, the acoustic cavity of the vehicle should be defined correctly in terms of its boundaries (VORLÄNDER, 2014). It is shown that the additional cavities strongly affect the total acoustic response. KANG et al. (2000) proposed a theoretical model to design the trim airgap system to improve the acoustic response of a passenger vehicle in the low frequency range. AHN et al. (2005) experimentally found an unexpected acoustic mode which is generated by the acoustic interaction between the cabin cavity and the trunk cavity. The air confined inside the cavities is excited by the panels, causing oscillations and creating high impedance. Under load, the confined air inside the cavities stores the potential energy. The air in the aperture that connects the trunk cavity and the cabin cavity possesses most of the kinetic energy.
In follow-up studies, the interaction between the cabin cavity and the trunk cavity is investigated. Pietrzyk, Bengtsson (2007) designed experiments and performed computational analysis to reveal out the effect of gaps on the parcel shelf of a passenger vehicle. LeE, LeE (2007a; 2007b) investigated the acoustic coupling between the adjacent cavities connected by necks. They measured and compared the frequency response functions for different cross-sectional areas and positions of a neck connecting adjacent cavities. LEE et al. (2011) proposed a complex acoustic finite element model which includes the cabin cavity and the trunk cavity, as well as equivalent-acoustic finite element models of the front and rear seats, parcel shelf, door volumes, instrument panel and HVAC volumes, and trunk wheel well volume. SEIFZADEH et al. (2014) experimentally studied the acoustic coupling of the cabin cavity and the trunk cavity on a full vehicle. OKTAV et al. (2016) proposed an analytical solution to calculate the planar acoustic eigenfrequencies of a passenger vehicle where the cabin cavity and the trunk cavity are connected through the folding rear-seat aperture.

As a countermeasure to trunk lid slam noise, airgaps are often located at the bottom corners of the 
trunk cavities of passenger vehicles. (The bottom corners of the cavity are chosen to locate the air-gaps, since the sound pressure reaches a maximum value at these locations.) These air-gaps open directly to air, if the reactive covers of them are ignored. To ignore the cover is reasonable, since it is made of plastics and textile, not the sheet metal. The current study suggests considering the air-gaps present in the trunk volume, while modelling the acoustic cavity of the computational model to be used in vibro-acoustic analysis. Since the trunk cavity and the cabin cavity are connected, the air-gaps present in the trunk affect the total acoustic response, even though they are not designed for this purpose. In brief, the air-gaps present in the trunk cavity behave like Helmholtz resonators, as they are open to air.

Helmholtz resonators were extensively studied by INGARD (1953). He examined the effect of different aperture geometries on the resonance frequency of resonators. AlSTER (1972) improved the calculation of resonant frequencies of Helmholtz resonators. SELAMET, RADAVICH (1997) investigated the acoustic performance and the effect of the specific cavity dimensions of resonators theoretically and experimentally. Li, Cheng (2007) suggested a general model for dealing with acoustic coupling between an enclosure and a Helmholtz resonator array. NAIR et al. (2010) experimentally investigated the coupled resonator-cavity systems. KOMKIN et al. (2014) estimated the eigenfrequency of a Helmholtz resonator fixed at the wall of a rectangular duct. Using the substructuring principle, YU et al. (2014) proposed a framework to deal with complex vibro-acoustic systems involving open acoustic cavities. Chintapalli, Padmanabhan (2015) derived a new end correction formula, for the orifice lengths of multi-orifice resonators.

The current study is organised as follows. In Sec. 2, two different simplified acoustic cavities are modelled, where the trunk cavity and the cabin cavity are coupled acoustically. The first one considers only the aperture between these cavities, whereas the second one counts on the air-gaps present in the trunk, as well. The proposed analytical solution is derived in Sec. 3. Using the analytical solution, the planar acoustic eigenfrequencies of the simplified model can be calculated. The computational and analytical results are compared in Sec. 4. Finally, brief conclusions are deduced.

\section{Modelling the acoustic cavity}

\subsection{Simplified geometry}

A rectangular geometry is constructed by considering the actual dimensions of acoustic cavity of the passenger vehicle (see Fig. 1). Regarding the general framework of vibro-acoustic studies, it is known that in

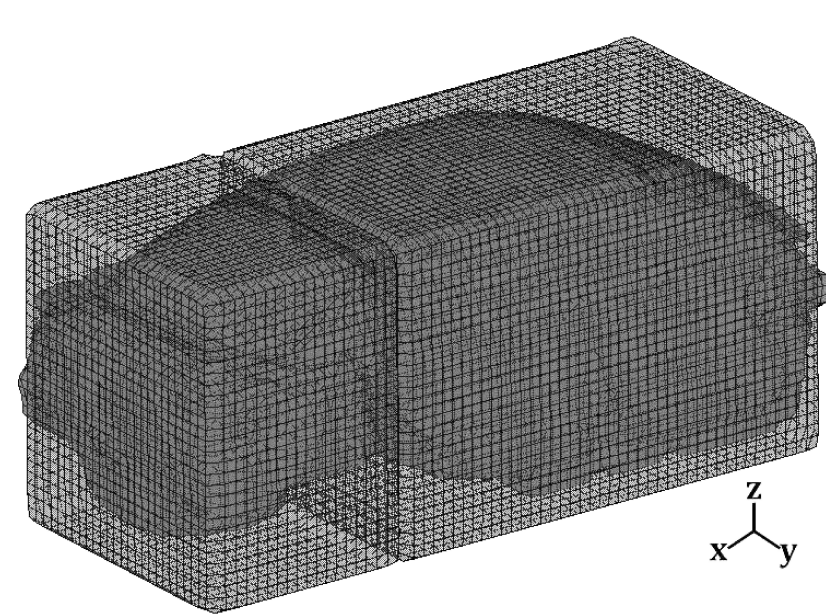

Fig. 1. Rectangular geometry is constructed according to the dimensions of the acoustic cavity of the passenger vehicle.

the low frequency range, structure-borne noise is dominant in vehicles (Shin, HASHIMOTO, 2013; Z̆IARAN, Chlebo, 2016). For this reason, the air-gaps present in the trunk cavity and the aperture between the trunk and cabin cavities are considered in the simplified geometry. The images of two air-gaps which are located symmetrically at the bottom corners of the trunk volume are shown in Fig. 2.

a)

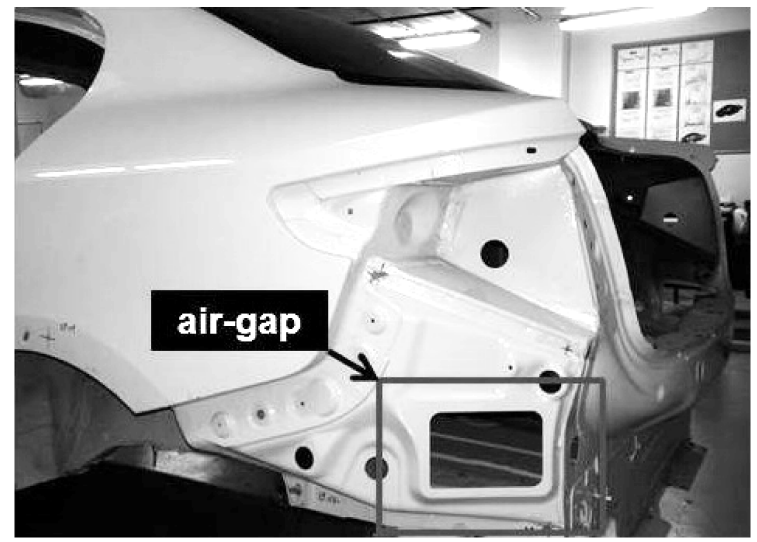

b)

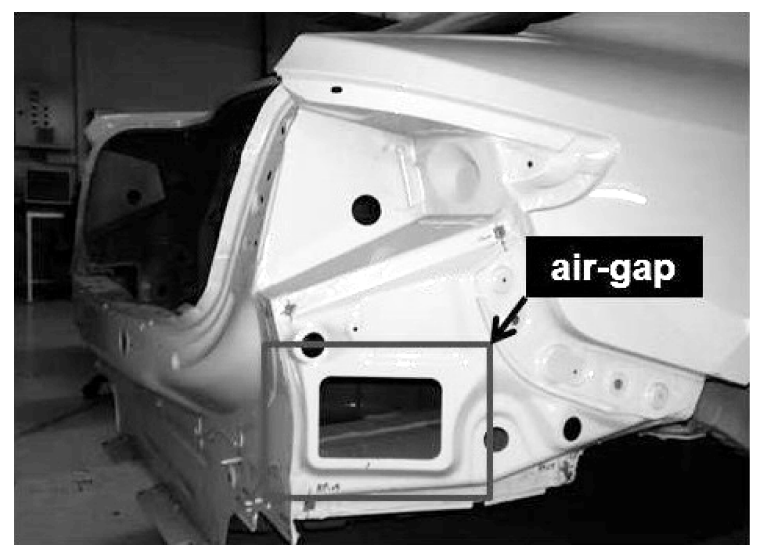

Fig. 2. Air-gaps present in the trunk volume: a) view from the left, b) view from the right. 
It is assumed that the eigenfrequencies of the actual cavity can be calculated approximately using its simplified geometry. It is shown that this assumption holds in the low frequency bandwidth, namely below $250 \mathrm{~Hz}$ (Xu et al., 2014; OKTAV et al., 2016).

Two different simplified geometries are constructed as shown in Fig. 3. In both of the models, the trunk and cabin cavities are modelled as two adjacent boxes connected by an aperture. In Model 1, only the aperture is considered, while in Model 2 the air-gaps present in the trunk are also taken into account.

a)

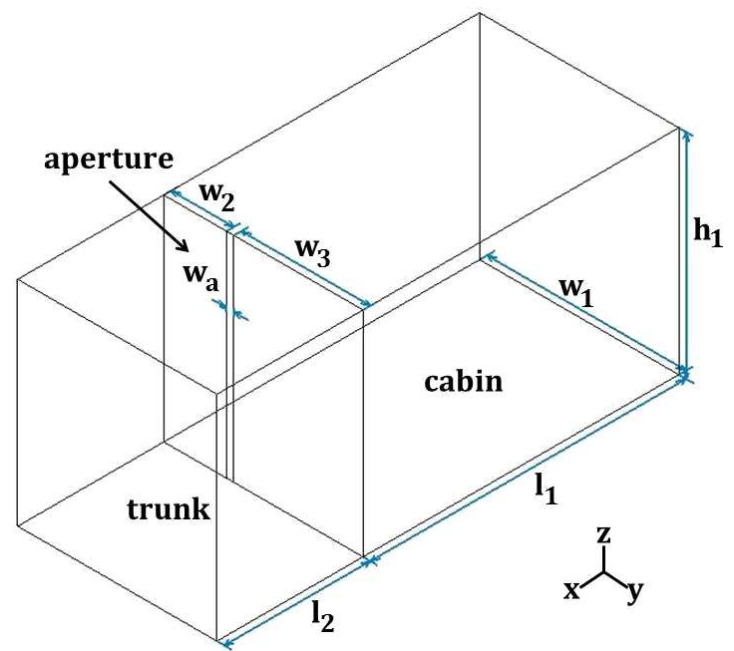

b)

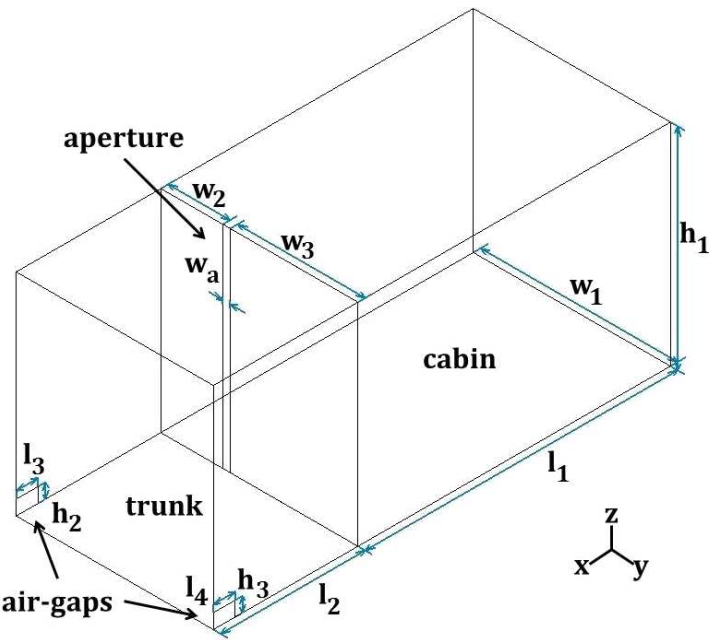

Fig. 3. Two simplified models of the acoustic cavity of the passenger vehicle: a) Model 1, b) Model 2.

\subsection{Finite element model of the simplified geometry}

The finite element models of the simplified geometries shown in Fig. 3 are constructed. The dimensions of the geometries are tabulated in Table 1. The acoustic cavities of the models are extracted using a commercial package. Considering the dimensions of models and the frequency bandwidth of interest, 530,622 HEXA8 type acoustical elements are constructed. In the acoustic analysis, the density of air and the speed
Table 1. Dimensions of the simplified geometries given in Fig. 3.

\begin{tabular}{|c|c|}
\hline Parameter & Size $[\mathrm{mm}]$ \\
\hline$l_{1}$ & 2200 \\
\hline$l_{2}$ & 1170 \\
\hline$l_{3}$ & 15 \\
\hline$l_{4}$ & 15 \\
\hline$w_{1}$ & 1300 \\
\hline$w_{2}$ & 455 \\
\hline$w_{3}$ & 915 \\
\hline$w_{a}$ & 30 \\
\hline$h_{1}$ & 1400 \\
\hline$h_{2}$ & 10 \\
\hline$h_{3}$ & 10 \\
\hline
\end{tabular}

of sound are taken as $1.20 \mathrm{~kg} / \mathrm{m}^{3}$ and $343 \mathrm{~m} / \mathrm{s}^{2}(\mathrm{~T}=$ $20^{\circ} \mathrm{C}$ ), respectively. The acoustic eigenfrequencies and mode shapes of the models are computed, where the analysis is limited up to $250 \mathrm{~Hz}$.

In Figs. 4 to 8, first five planar acoustic eigenfrequencies and the corresponding acoustic mode shapes of the two models are shown. It is observed that the air-gaps present in the trunk alter the acoustic mode shapes substantially.

a)

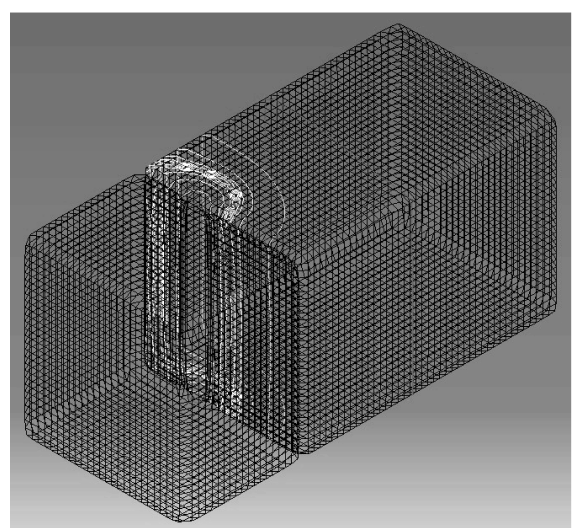

b)

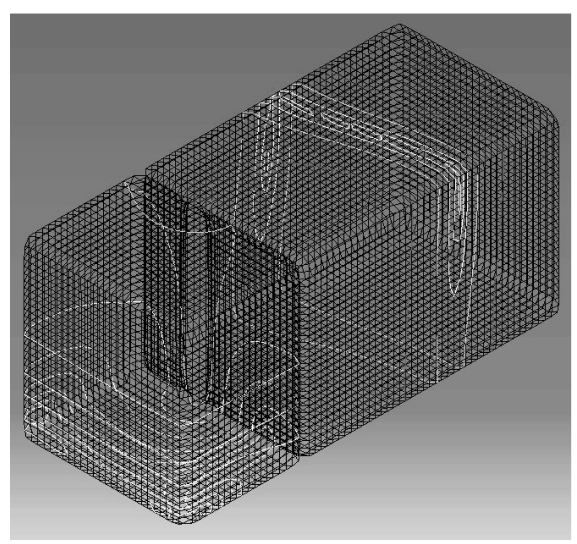

Fig. 4. Acoustic mode shapes of the simplified models at $41 \mathrm{~Hz}\left(\begin{array}{lll}1 & 0 & 0)\end{array}\right)$ a) Model 1, b) Model 2 . 
a)

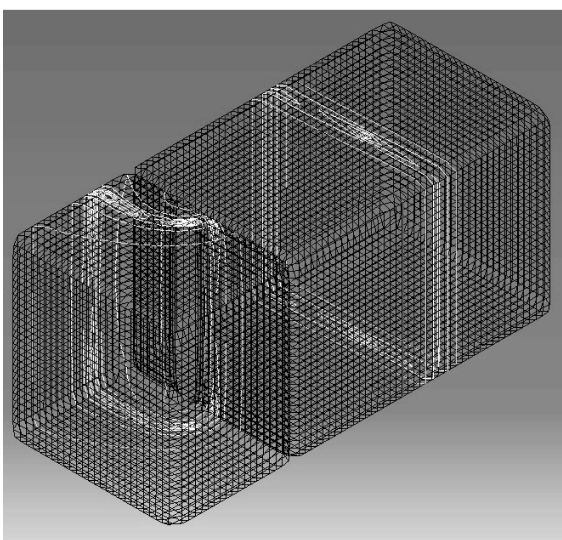

b)

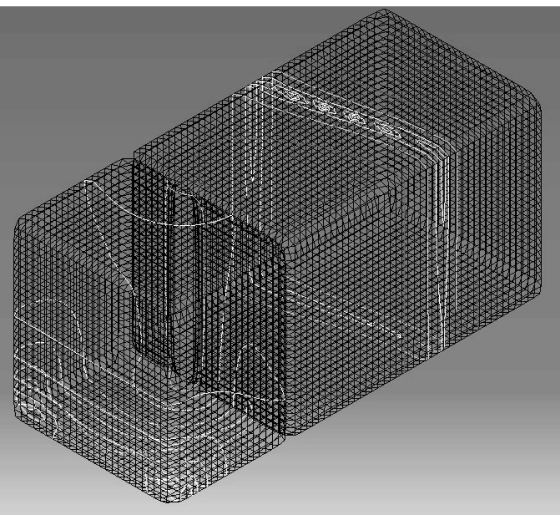

Fig. 5. Acoustic mode shapes of the simplified models at $89 \mathrm{~Hz}(2 \quad 0$ 0): a) Model 1, b) Model 2.

a)

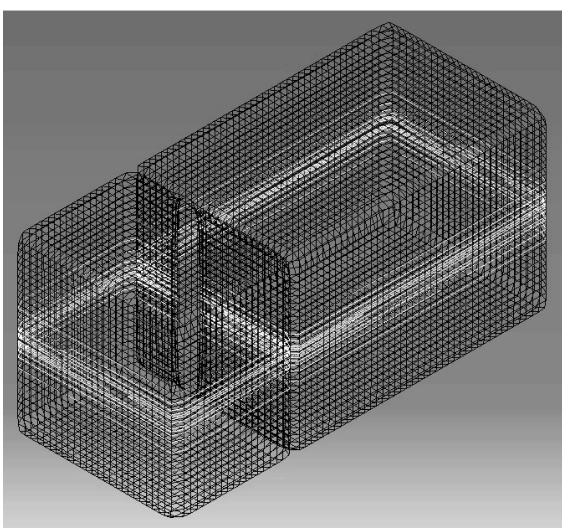

b)

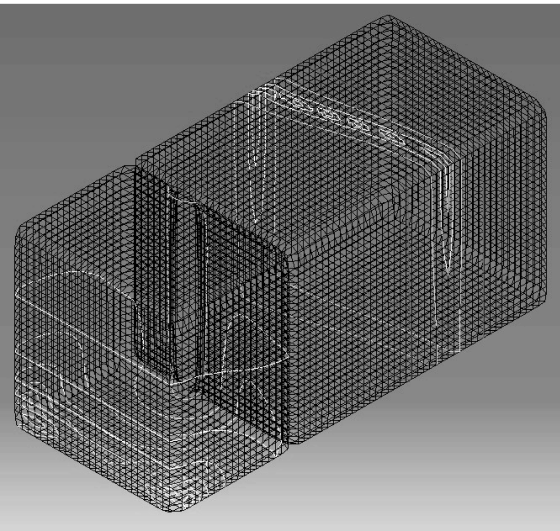

Fig. 6. Acoustic mode shapes of the simplified models at $122.5 \mathrm{~Hz}$ (0 0 1): a) Model 1, b) Model 2. a)

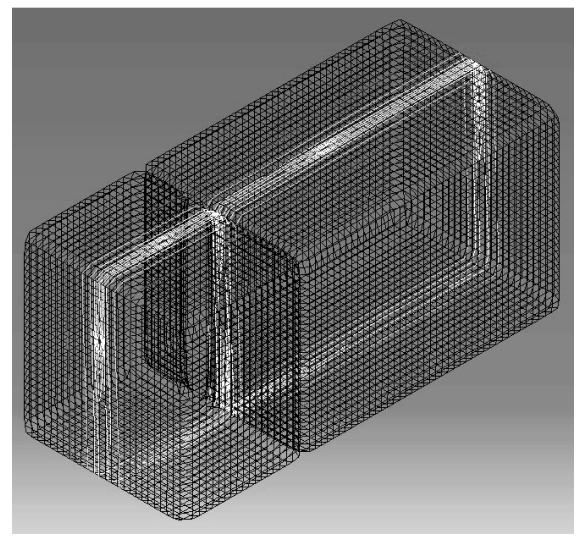

b)

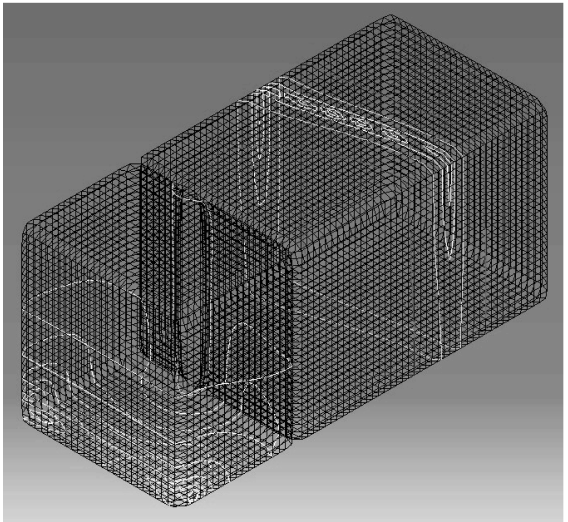

Fig. 7. Acoustic mode shapes of the simplified models at $132 \mathrm{~Hz}(010)$ : a) Model 1, b) Model 2 .

a)

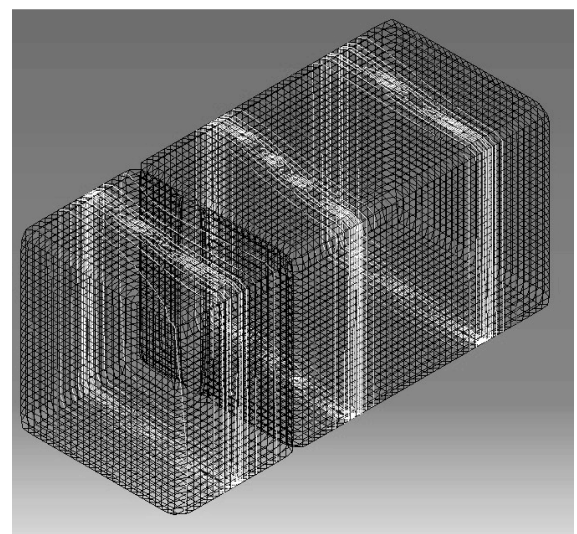

b)

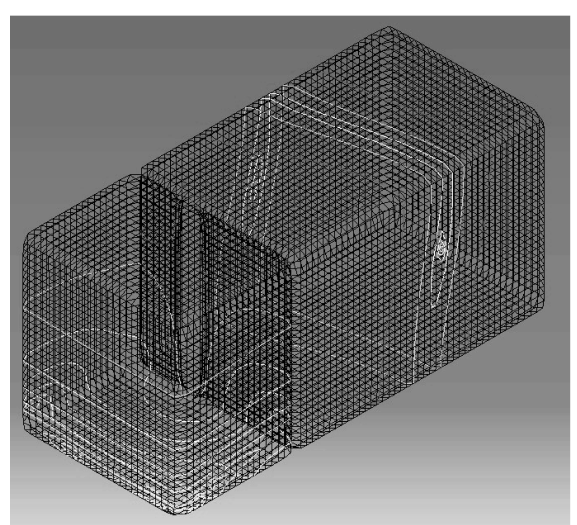

Fig. 8. Acoustic mode shapes of the simplified models at $162 \mathrm{~Hz}$ (3 0 0): a) Model 1, b) Model 2. 
The difference between the acoustic mode shapes of two models is one of the highlights of the current study. As will be discussed in more detail in Sec. 4, this situation alters the sound pressure levels perceived in the target microphone locations, which is obviously important in any vibro-acoustic framework. It could have been useful, if these air-gaps were specifically designed to control the acoustic response of the passenger vehicle, which is not the case. These air-gaps are designed as a countermeasure to trunk lid slam noise only, but they alter the acoustic response, since the trunk and cabin cavities interact. In the next section, an analytical solution is proposed to calculate the planar acoustic eigenfrequencies.

\section{Analytical solution}

When there is no ambient flow, the reduced wave equation for complex sound pressure amplitude is given by

$$
\nabla^{2} p+(\omega / c)^{2} p=0
$$

Herein, pressure $p$ is the dependent field variable, and $c$ is the speed of sound. The solution of Eq. (1) results in so-called standing waves, provided that all of the walls of a rectangular box are assumed to be rigid (MarburG, Nolte, 2008). Regarding the separation of variables, complex sound pressure amplitude for a rectangular box with dimensions $l, w$, and $h$ is

$$
p_{l m n}=A \cos \left(k_{x} x\right) \cos \left(k_{y} y\right) \cos \left(k_{z} z\right) \text {, }
$$

where

$$
k_{x}=j \pi / l, \quad k_{y}=m \pi / w, \quad k_{z}=n \pi / h .
$$

Herein, $j, m, n$ are integers. For a regular rectangular cavity, the analytical solution expressed by Eq. (2) can be used in the computation of acoustic eigenfrequencies. However, the geometry shown in Fig. 3a is not a regular rectangular cavity, it has a partition that separates the two acoustic cavities. For an analytical solution, it is assumed that the aperture on the partition is replaced by a rectangular piston in the same dimensions. This fictitious piston moves to and fro in the $x$-direction with a uniform velocity amplitude $v_{p}$, as suggested by INGARD (1953). It is assumed that the piston is rigid and the participation of the piston to the velocity field is limited to the $x$-direction, only. The width and height of the piston are $w_{a}$ and $h_{a}$, respectively. The average velocity of the piston can be expressed as

$$
v_{0}=\frac{w_{a} h_{a}}{w_{1} h_{1}} v_{p}
$$

In that case, the acoustic pressure is

$$
p(x, \omega)=A \cos \left(k_{y} y\right) \cos \left(k_{z} z\right) \mathrm{e}^{\mathrm{i} k_{x} x}
$$

where $k_{y}$ and $k_{z}$ are identical with those in Eq. (3). Acoustic pressure is uniform in the fundamental mode $\left(p_{0}\right)$, which allows to express the following:

$$
k_{x}=k=\omega / c \text {. }
$$

In the fundamental mode, the axial system and the average piston velocities are equal. Hence, the pressure amplitude is

$$
p_{0}=\rho c \frac{w_{a} h_{a}}{w_{1} h_{1}} v_{p} \mathrm{e}^{\mathrm{i} k x}
$$

The normalised reactance of the piston is given as

$$
\chi=\frac{\mathrm{i} \omega M}{w_{a} h_{a} \rho c} \equiv \mathrm{i} k \varphi,
$$

where $M$ is the mass load on the piston. The acoustic radiation impedance of the piston is expressed by

$$
Z_{a}=\frac{p_{0}}{\rho c v_{p}}=\frac{w_{a} h_{a}}{w_{1} h_{1}}-\mathrm{i} k \varphi .
$$

Herein, $\mathrm{i} k \varphi$ is the mass reactance (INGARD, 2010). The physical interpretation for the reactance length is that $\varphi$ can be taken as the length of air column in the aperture. Observing the simplified geometry given in Fig. 3a, it is assumed that the reactance length $(\varphi)$ is effective only in the $x$-direction. Henceforth, the problem can be analysed in two parts:

1. The acoustic eigenfrequencies of planar modes in the $y$ - and $z$-directions can be calculated using Eqs. (2) and (3).

2. The acoustic eigenfrequencies of planar modes in the $x$-direction can be calculated using the analytical solution outlined below, where the reactance length is considered.

The mentioned analytical solution requires the computation of the reactance length for higher orders, as well. Note that, in the low frequency bandwidth, where $k_{x} w_{a} \ll 1$ is valid, the reactance length depends only on the dimensions of geometry, rather than the frequency. Hence, the axial velocity field is expressed by

$$
v_{x}=\sum_{m n=0}^{\infty} \frac{k_{x}}{k} \frac{p_{m n}}{\rho c} \cos \left(k_{y} y\right) \cos \left(k_{z} z\right) \mathrm{e}^{\mathrm{i} k x} .
$$

Using orthogonality $(m \neq n)$ and integrating over the area of the aperture,

$$
\begin{aligned}
v_{m n} & =\iint_{\text {aperture }} v_{0} \cos \left(k_{y} y\right) \cos \left(k_{z} z\right) \mathrm{d} y \mathrm{~d} z \\
& =v_{0} \frac{w_{a}}{w_{1}} \frac{h_{a}}{h_{1}} \gamma_{m n} \frac{\sin \left(k_{y} w_{a}\right)}{k_{y} w_{a}} \frac{\sin \left(k_{z} h_{a}\right)}{k_{z} h_{a}}, \\
m, n & >0,
\end{aligned}
$$


where $\gamma_{0,0}=1, \gamma_{m, 0}=\gamma_{0, n}=2$, and $\gamma_{m, n}=4$. The velocity distribution at the partition $x=x^{\prime}$ is

$$
v_{x^{\prime}}=\sum_{m n}^{\infty} v_{m n} \cos \left(k_{y} y\right) \cos \left(k_{z} z\right)
$$

The velocities expressed in Eqs. (10) and (12) should be equal at the partition $\left(x=x^{\prime}\right)$, hence,

$$
p_{m n}=\rho c v_{m n} \frac{k}{k_{x}} \text {. }
$$

Below the cut off frequency, where $k_{x} \simeq k_{m n}$, the transverse contribution $p_{m n}$ corresponds to the mass reactance part of Eq. (9). Thus, the reactance length is expressed by

$$
\varphi=\sum_{m n}^{\infty} \frac{1}{k_{m n}} \frac{w_{a}}{w_{1}} \frac{h_{a}}{h_{1}} \gamma_{m n} \frac{\sin \left(k_{y} w_{a}\right)}{k_{y} w_{a}} \frac{\sin \left(k_{z} h_{a}\right)}{k_{z} h_{a}} .
$$

The reactance length $(\varphi)$ is contributed by all transverse modes $(m, n)$ except the fundamental mode ( $m=n=0$ ). Hence, it is excluded from the summation in Eq. (14), where a star symbol is used for distinction. Defining the ratio of widths as $\xi=w_{a} / w_{1}$ and the ratio of heights as $\zeta=h_{a} / h_{1}$, the reactance length can be expressed as

$$
\varphi=\sum_{m n}^{\infty} \frac{\xi \zeta}{k_{m n}} \gamma_{m n} \frac{\sin (m \pi \xi) \sin (n \pi \zeta)}{m n \xi \zeta \pi^{2}} .
$$

At the beginning of the derivation, it is assumed that the air inside the cavities is driven by the piston located instead of aperture, see Fig. 3a. As the piston moves to and fro, plane waves will form in both of the cavities (PAnton, Miller, 1975). Regarding the plane waves formed in $+x$ and $-x$ directions, acoustic impedance can be written as

$$
Z_{a}=\frac{\rho c}{w_{1} h_{1}} \frac{\alpha \mathrm{e}^{\mathrm{i} k_{x} x}+\beta \mathrm{e}^{-\mathrm{i} k_{x} x}}{\alpha \mathrm{e}^{\mathrm{i} k_{x} x}-\beta \mathrm{e}^{-\mathrm{i} k_{x} x}} .
$$

In theory, the acoustic impedance is infinite at $x=l_{1}$ and $x=l_{2}$, which in turn yields

$$
Z_{a}=\frac{\rho c}{\mathrm{i} w_{1} h_{1}} \cot \left(k_{x} l_{n}\right)
$$

where $n=1,2$. The aperture possesses most of the kinetic energy of the system (HuANG, 2000). Thus, it can be said that at $x=x^{\prime}$ the impedance is purely reactive. In that case,

$$
Z_{a}=\mathrm{i} \omega \frac{M}{w_{a}^{2} h_{a}^{2}}
$$

Considering Eqs. (8) and (18), the acoustic impedance can be written as

$$
Z_{a}=\mathrm{i} \frac{\varphi \rho c k_{x}}{w_{a} h_{a}}
$$

Substitution of Eq. (19) into Eq. (17) results in the following transcendental equation:

$$
\frac{\varphi k_{x}}{\xi \zeta}=\cot \left(k_{x} l_{1}\right)+\cot \left(k_{x} l_{2}\right)
$$

which can be used to calculate the higher orders of $k_{x}$ for the simplified geometry given in Fig. 3a.

\section{Computational and analytical results}

\subsection{Comparison of eigenfrequency values}

Using Eqs. (15) and (20), higher orders of $k_{x}$ are calculated (see Fig. 9). Next, acoustic eigenfrequencies in the $x$-direction are calculated by

$$
f_{j}=c k_{x} / 2 \pi
$$

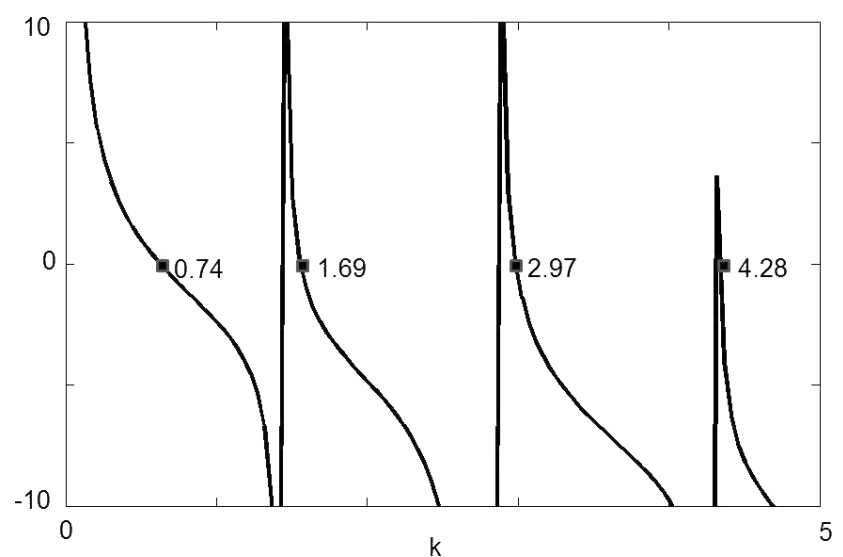

Fig. 9. Numerical solution of Eq. (20): first four values of the wave number $k_{x}$.

As explained in the preceding section, the problem is analysed in two parts. The planar acoustic eigenfrequencies in $y$ - and $z$-directions are calculated employing Eq. (2) and (3), whereas the ones in the $x$ direction are calculated using Eq. (21). The computational and analytical results are tabulated in Table 2. Note, that the convergence of double summation stated in Eq. (15) is fast, i.e. a number of 100 transverse modes is often enough.

Table 2. Comparison of the computational and analytical eigenfrequencies of the simplified model (results are in $\mathrm{Hz}$ ).

\begin{tabular}{|c|c|c|}
\hline Acoustic mode & Computational & Analytical \\
\hline$\left(\begin{array}{lll}1 & 0 & 0\end{array}\right)$ & 40.9 & 40.4 \\
\hline$\left(\begin{array}{lll}2 & 0 & 0\end{array}\right)$ & 89.0 & 92.2 \\
\hline$\left(\begin{array}{lll}0 & 0 & 1\end{array}\right)$ & 122.5 & 122.5 \\
\hline$\left(\begin{array}{lll}0 & 1 & 0\end{array}\right)$ & 132.0 & 132.0 \\
\hline$\left(\begin{array}{lll}3 & 0 & 0\end{array}\right)$ & 162.3 & 162.4 \\
\hline
\end{tabular}




\subsection{Effect of air-gaps on the SPL values}

A forced vibration analysis is conducted to reveal the effect of air-gaps on the sound pressure level computed in the target microphone locations. The acoustic cavity is excited through a plate located in the front face of the simplified model, as shown in Fig. 10. The plate is subjected to an external harmonic force located on the upper corner. The magnitude of force is $1 \mathrm{~N}$.

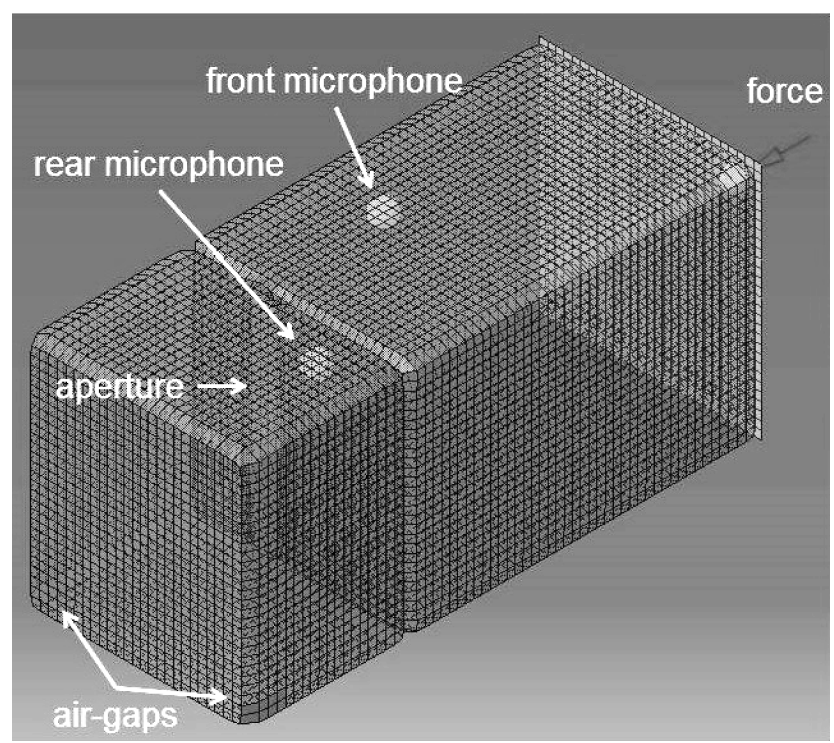

Fig. 10. Forced vibration analysis of the system.

Two microphone locations are determined in the model. These locations essentially correspond to the actual microphone locations used during the track test of the real passenger vehicle. The front microphone is employed to measure the sound pressure level (SPL) perceived at the left ear level of the driver, and the rear microphone is used to measure the SPL perceived by the rear seat occupants. The computed SPLs are given in Figs. 11 and 12, where the acoustic responses of Model 1 and Model 2 are compared. Substantial

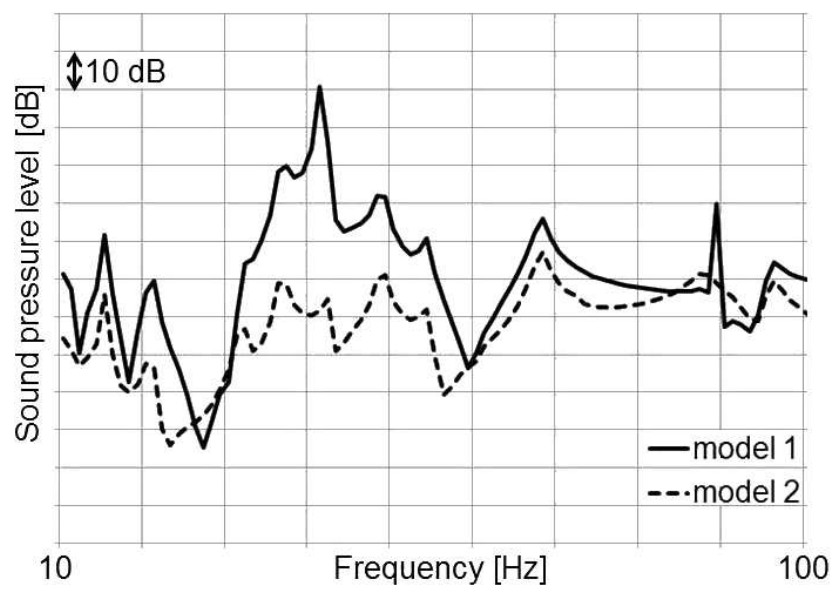

Fig. 11. Sound pressure levels computed at the front microphone level.

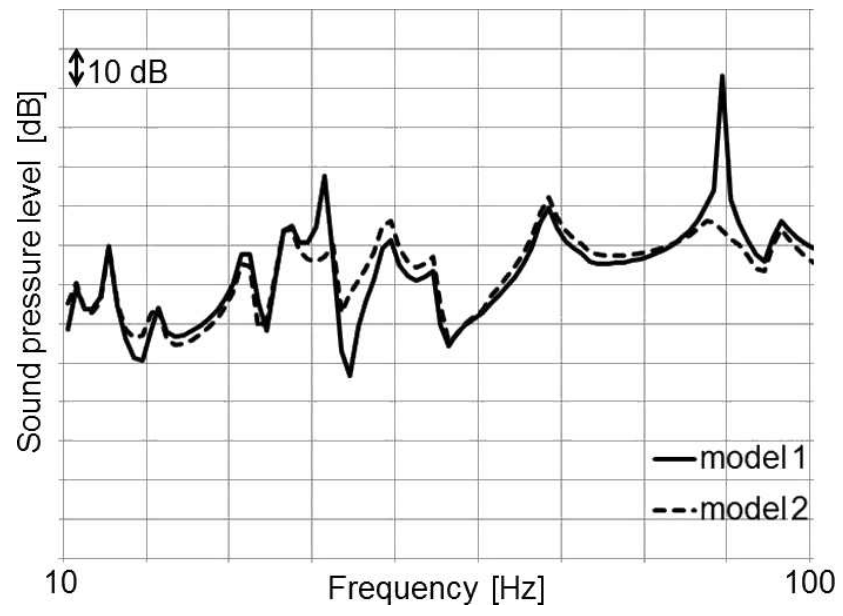

Fig. 12. Sound pressure levels computed at the rear microphone level.

differences observed in the acoustic responses of two models also given idea about improving the acoustic response. It is observed that the air-gaps can be used to enhance the acoustic characteristics of the passenger vehicle, provided their geometries are also designed for this purpose, beside their countermeasure functionality against the trunk lid slam noise.

\section{Conclusion}

In this work, the effects of trunk cavity and airgaps in the acoustic response of a passenger vehicle are studied. Several observations are made:

- The trunk cavity and the cabin cavity are acoustically coupled through an aperture in the folding rear-seat.

- The air-gaps used against the trunk lid slam noise behave as Helmholtz resonators.

- The trunk cavity and the air-gaps present in the trunk affect the acoustic response of the passenger vehicle.

An analytical solution is derived to calculate the wave number $\left(k_{x}\right)$ in the $x$-direction. It is shown that the proposed analytical procedure is effective in the calculation of the planar acoustic eigenfrequencies. Further, the effect of air-gaps on the acoustic modes is shown using computational models. The deviations in the sound pressure levels are also shown through a generic case study. It is concluded that the air-gaps present in the trunk cavity can be also considered to improve the acoustic response of passenger vehicles.

\section{References}

1. Ahn C.G., Choi H.G., Lee J.M. (2005), Structuralacoustic coupling analysis of two cavities connected by boundary structures and small holes, Journal of Vibration and Acoustics, 127, 6, 566-574. 
2. Alster M. (1972), Improved calculation of resonant frequencies of Helmholtz resonators, Journal of Sound and Vibration, 24, 1, 63-85.

3. Chintapalli V.R., Padmanabhan C. (2015), Design relations and simplified reactance formulas for multiorifice Helmholtz resonators, Acta Acustica united with Acustica, 101, 1, 124-133.

4. Ferreira T.S., Magalhães P.A., Moura F.L., FERREIRA T.S. (2016), The Effect of the Cavity Damping on Vehicular Evaluation using the Finite Element Method, Archives of Acoustics, 41, 1, 87-97.

5. Huang L. (2000), A theory of reactive control of lowfrequency duct noise, Journal of Sound and Vibration, 238, 4, 575-594.

6. Ingard U. (1953), On the theory and design of acoustic resonators, The Journal of the Acoustical Society of America, 25, 6, 1037-1061.

7. Ingard U. (2010), Notes on Acoustics, Laxmi Publications Ltd.

8. Kang S.W., LeE J.M., Kim S.H. (2000), Structuralacoustic coupling analysis of the vehicle passenger compartment with the roof, air-gap, and trim boundary, Journal of Vibration and Acoustics, 122, 3, 196-202.

9. Komkin A.I., Mironov M.A., Yudin S.I. (2014), Eigenfrequency of a Helmholtz resonator at the wall of a rectangular duct, Acoustical Physics, 60, 2, 142-147.

10. LeE J.W., LeE J.M. (2007a), Forced vibro-acoustical analysis for a theoretical model of a passenger compartment with a trunk - Part I: Theoretical part, Journal of Sound and Vibration, 299, 4, 900-917.

11. LeE J.W., LEE J.M. (2007b), Forced vibro-acoustical analysis for a theoretical model of a passenger compartment with a trunk - Part II: Experimental part, Journal of Sound and Vibration, 299, 4, 918-932.

12. Lee S., Park K., Sung S.H., Nefske D.J. (2011), Boundary condition effect on the correlation of an acoustic finite element passenger compartment model, SAE International Journal of Materials and Manufacturing, 4, 1, 708-715.

13. Li D., Cheng L. (2007), Acoustically coupled model of an enclosure and a Helmholtz resonator array, Journal of Sound and Vibration, 305, 1, 272-288.

14. Marburg S., Nolte B. (2008), Computational Acoustics of Noise Propagation in Fluids: Finite and Boundary Element Methods, vol. 578, Berlin, Heidelberg: Springer.
15. Nair S.U., Shete C.D., Subramoniam A., HanDoo K.L., Padmanabhan C. (2010), Experimental and computational investigation of coupled resonator-cavity systems, Applied Acoustics, 71, 1, 61-67.

16. Oktav A., Anlaş G., Yilmaz Ç. (2016), The effect of the folding rear-seat aperture in the acoustic response of a sedan car, Proceedings of the Institution of Mechanical Engineers, Part D: Journal of Automobile Engineering, 231, 2, 253-266.

17. Panton R.L., Miller J.M. (1975), Resonant frequencies of cylindrical Helmholtz resonators, The Journal of the Acoustical Society of America, 57, 6, 1533-1535.

18. Pietrzyk A., Bengtsson T. (2007), An investigation of the coupling between the passenger compartment and the trunk in a sedan, SAE Technical Paper, No. 200701-2356.

19. Seifzadeh A., Pietrzyk A., Göransson P., RAMAKRISHNAN R. (2014), Effect of coupling between passenger compartment and trunk of a car on coupled system natural frequencies using acoustic frequency response function, Applied Acoustics, 76, 310-318.

20. Selamet A., Radavich P.M. (1997), The effect of length on the acoustic attenuation performance of concentric expansion chambers: an analytical, computational and experimental investigation, Journal of Sound and Vibration, 201, 4, 407-426.

21. Shin S.H., Hashimoto T. (2013), Optimum order spectrum profiles for improvement of sound quality of car interior noise, Noise Control Engineering Journal, 61, 6, 578-589.

22. VORLÄNDER M. (2014), Virtual acoustics, Archives of Acoustics, 39, 3, 307-318.

23. Xu H., Dickinson O., Wang J., Kang H. (2014), Acoustic cavity modal analysis for NVH development of road machinery cabins, Topics in Modal Analysis II, 8, Springer International Publishing.

24. Yu X., Cheng L., Guyader J.L. (2014), Modeling vibroacoustic systems involving cascade open cavities and micro-perforated panels, The Journal of the Acoustical Society of America, 136, 2, 659-670.

25. Z̆iaran S., Chlebo O. (2016), Noise control transmission methods of the combustion engine by means of reduction of the vibration, Archives of Acoustics, 41, 2, $277-284$. 